\title{
Optimal additive quaternary codes of low dimension
}

\author{
Jürgen Bierbrauer \\ Department of Mathematical Sciences \\ Michigan Technological University \\ Houghton, Michigan 49931 (USA) \\ S. Marcugini* and F. Pambianco* \\ Dipartimento di Matematica e Informatica \\ Università degli Studi di Perugia \\ Perugia (Italy)
}

July 13, 2020

\begin{abstract}
An additive quaternary $[n, k, d]$-code (length $n$, quaternary dimension $k$, minimum distance $d$ ) is a $2 k$-dimensional $\mathbb{F}_{2}$-vector space of $n$-tuples with entries in $Z_{2} \times Z_{2}$ (the 2-dimensional vector space over $\mathbb{F}_{2}$ ) with minimum Hamming distance $d$. We determine the optimal parameters of additive quaternary codes of dimension $k \leq 3$. The most challenging case is dimension $k=2.5$. We prove that an additive quaternary $[n, 2.5, d]$-code where $d<n-1$ exists if and only if $3(n-d) \geq\lceil d / 2\rceil+\lceil d / 4\rceil+\lceil d / 8\rceil$. In particular we construct new optimal 2.5-dimensional additive quaternary codes. As a by-product we give a direct proof for the fact that a binary linear
\end{abstract}

*The research of S. Marcugini and F. Pambianco was supported in part by the Italian National Group for Algebraic and Geometric Structures and their Applications (GNSAGA - INDAM) and by University of Perugia (Project: Curve, codici e configurazioni di punti, Base Research Fund 2018). 
$[3 m, 5,2 e]_{2}$-code for $e<m-1$ exists if and only if the Griesmer bound $3(m-e) \geq\lceil e / 2\rceil+\lceil e / 4\rceil+\lceil e / 8\rceil$ is satisfied.

Keywords: Quaternary additive codes, projective spaces, optimal codes, binary linear codes.

\section{Introduction}

The concept of additive codes is a far-reaching and natural generalization of linear codes, see [2], Chapter 18. Here we restrict to the quaternary case.

Definition 1. Let $k$ be such that $2 k$ is a positive integer. An additive quaternary $[n, k]$-code $C$ (length $n$, dimension $k$ ) is a $2 k$-dimensional subspace of $\mathbb{F}_{2}^{2 n}$ where the coordinates come in pairs of two. We view the codewords as $n$ tuples where the coordinate entries are elements of $\mathbb{F}_{2}^{2}$ and use the Hamming distance.

We write the parameters of the code as $[n, k, d]$ where $d$ is the minimum Hamming distance. Here $k$ is the quaternary dimension. As an example, in case $k=2.5$ the code is a 5 -dimensional vector space over $\mathbb{F}_{2}$. Additive codes are particularly interesting because of a link to quantum stabilizer codes, see [4, 5, 9]. We will also use the geometric construction of additive quaternary codes. In fact, a quaternary $[n, k, d]$-code is equivalent to a multiset of $n$ lines in $P G(2 k-1,2)$ such that each hyperplane of $P G(2 k-1,2)$ contains at most $s=n-d$ of those lines, in the multiset sense. Blokhuis and Brouwer [8] first suggested the problem of determining the optimum parameters of additive quaternary codes. In earlier work we determined all such optimal parameters when $n \leq 13$, see [2], Chapter 18 and [6]. For further results concerning larger lengths see [1, 7]. In the present work we determine all optimal parameters when the quaternary dimension is $k \leq 3$. Dimensions $k \leq 2$ are degenerate cases, see Section 2, Dimension 3 is easily dealt with as well, see Section 3. Our main result is Theorem 2 in Section 4 where the optimal parameters of 2.5-dimensional additive quaternary codes are determined. For $k>1$ we prefer to work with the species $s=n-d$ instead of the minimum distance $d$. Define $n_{k}(s)$ to be the maximal length $n$ such that an additive $[n, k, n-s]$-code exists. For integer $k$, let $n_{k, \text { lin }}(s)$ be the maximal $n$ such that a linear quaternary $[n, k, n-s]_{4}$-code exists. In the present paper we determine $n_{k}(s)$ for $k \leq 3$ and all $s$. The following obvious lemma will be used to prove nonexistence results: 
Lemma 1. The concatenation of a quaternary additive $[n, k, d]$-code and the binary linear $[3,2,2]_{2}$-code is a binary linear $[3 n, 2 k, 2 d]_{2}$-code.

\section{Dimensions $k \leq 2$.}

Clearly dimension $k=1$ is a trivial case, the optimal parameters being $[n, 1, n]$. Dimension $k=1.5$ is degenerate as well. The ambient space is the Fano plane and the optimal choice is to use each of its seven lines with multiplicity $s$. This shows $n_{1.5}(s)=7 s$. The corresponding codes have parameters $[7 s, 1.5,6 s]$. Dimension $k=2$ still is degenerate. In the linear case we have $n_{2, \text { lin }}(s)=5 s$. In fact we work in the projective line $P G(1,4)$ and the optimal choice is to use each of its points with multiplicity $s$.

Proposition 1. We have $n_{2}(s)=n_{2, \text { lin }}(s)=5 s$ for all $s$.

Proof. Assume $n_{2}(s)>5 s$. We would have a $[5 s+1,2,4 s+1]$-code. Lemma 1 would yield a binary linear $[15 s+3,4,8 s+2]_{2}$-code. This contradicts the Griesmer bound.

\section{The case of dimension $k=3$.}

The optimal parameters of linear quaternary 3-dimensional codes are of course known:

Proposition 2. We have $n_{3, \text { lin }}(2)=6, n_{3, \text { lin }}(3)=9, n_{3, \text { lin }}(4)=16$,

$n_{3, \text { lin }}(5 i)=21 i, n_{3, \text { lin }}(5 i+1)=21 i+1$ and $n_{3, \text { lin }}(5 i+\sigma)=21 i+1+5(\sigma-1)$

for $i \geq 1, \sigma \in\{2,3,4\}$.

Proof. For $d<9$ this is easy to check. For larger $d$ we can invoke a result by

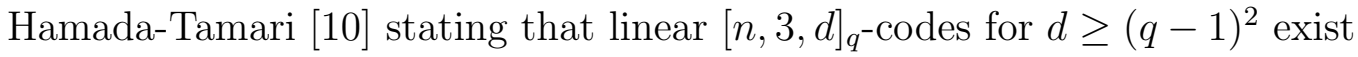
if and only if the parameters satisfy the Griesmer bound (see [2], Theorem 17.7). This coincides with the statement of our proposition.

Theorem 1. We have $n_{3}(s)=n_{3, \text { lin }}(s)$ for all $s$.

Proof. Assume there is an additive 3-dimensional code with larger $n$ and the same species. We illustrate with case $s=5 i$. We would have a $[21 i+$ $1,3,16 i+1]$-code. Lemma 1 yields a linear $[63 i+3,6,32 i+2]_{2}$-code, which contradicts the Griesmer bound. The other cases are analogous. 


\section{The case of dimension 2.5.}

Our main result is the following:

Theorem 2. An additive quaternary $[n, 2.5, d]$-code where $d<n-1$ exists if and only if $3(n-d) \geq\lceil d / 2\rceil+\lceil d / 4\rceil+\lceil d / 8\rceil$.

In the present section we prove Theorem 2. In the sequel use the abbreviation $d_{l}=\lceil d / l\rceil$. The necessity is obvious. In fact, Lemma 1 applied to an additive quaternary $[n, 2.5, d]$-code yields a binary linear $[3 n, 5,2 d]_{2}$-code. The condition of Theorem 2 is the Griesmer bound as applied to this binary code. It remains to prove sufficiency: given $d, n$ satisfying the condition of the theorem we need to construct an additive quaternary $[n, 2.5, d]$-code. As before, let $s=n-d$. For each $s$ consider the pair $D_{s}=\left(s, m_{s}\right)$ where $m_{s}$ is the maximal $n$ such that $n, d=n-s$ satisfy the condition in Theorem 2 . We need to prove the existence of an $\left[m_{s}, 2.5, m_{s}-s\right]$-code, for all $s \geq 2$. When such a code exists we say that we represented $D_{s}$. Here are some examples:

$D_{2}=(2,8), D_{3}=(3,11), D_{4}=(4,16), D_{5}=(5,21), D_{6}=(6,26), D_{7}=(7,31)$.

Let $C$ be an $[n, 2.5, d]$-code and $C^{\prime}$ the code obtained by increasing each line multiplicity of $C$ by 1 . As $P G(4,2)$ has 155 lines and $P G(3,2)$ has 35 lines we see that $C^{\prime}$ is an $[n+155,2.5, d+120]$-code. Concerning the bound of the theorem we observe that $3(n-d)-d_{2}-d_{4}-d_{8}$ is invariant under the substitution $n \mapsto n+155, d \mapsto d+120$. This shows that we need prove the existence of an $[n, 2.5, d]$-code only for $n<155$. This means that it suffices to construct $D_{2}, D_{3}, \ldots, D_{35}=(35,155)$. Observe that there is an obvious sum construction which shows that the existence of codes $\left[m_{1}, 2.5, l_{1}\right]$ and $\left[m_{2}, 2.5, l_{2}\right]$ implies the existence of an $\left[m_{1}+m_{2}, 2.5, l_{1}+l_{2}\right]$-code. This shows that if $D_{s_{1}}$ and $D_{s_{2}}$ can be constructed then also $D_{s_{1}}+D_{s_{2}}$ can be constructed. We see now that it suffices to construct $D_{2}, \ldots, D_{7}$ as the remaining $D_{s}, s \leq 35$ follow from the sum construction. Here are some examples:

$D_{8}=D_{6}+D_{2}, D_{9}=D_{7}+D_{2}, D_{10}=D_{5}+D_{5}, D_{11}=D_{9}+D_{2}, D_{12}=D_{6}+D_{6}$.

It remains to construct $D_{2}, \ldots, D_{7}$. Now $D_{2}$ implies $D_{4}$ as $D_{2}+D_{2}=D_{4}$ and $D_{5}=(5,21)$ is constructed as there is even a linear $[21,3,16]_{4}$-code (corresponding to the points of $P G(2,4)$ ). We are reduced to construct $D_{2}, D_{3}, D_{6}, D_{7}$. Now $D_{2}=(2,8)$ corresponds to a $[8,2.5,6]$-code. This is 
the Blokhuis-Brouwer construction [8, 3]. In the same context an $[11,2.5,8]$ code was constructed. This is a representation of $D_{3}=(3,11)$. We are finally reduced to construct $D_{6}$ and $D_{7}$.

\section{A construction}

Consider a chain

$$
l_{0} \subset E_{0} \subset H_{0} \subset P G(4,2)
$$

where $l_{0}$ is a line, $E_{0}$ a plane and $H_{0}$ a solid (hyperplane) in $P G(4,2)$. Let $\mathcal{V}$ be a set of 8 lines such that each point in $E_{0} \backslash l_{0}$ is on precisely two lines of $\mathcal{V}$, each point outside $H_{0}$ is on precisely one line of $\mathcal{V}$. Also, let $\mathcal{E}$ be a set of 8 lines partitioning the points outside $E_{0}$ (Blokhuis-Brouwer construction).

Definition 2. Let $C(g, h, v, e)$ be the additive 2.5-dimensional quaternary code described by the following multiset of lines: line $l_{0}$ with multiplicity $g$, the remaining lines of $E_{0}$ each with multiplicity $h$, the lines of $\mathcal{V}$ with multiplicity $v$ and the lines of $\mathcal{E}$ with multiplicity $e$.

Clearly $C(g, h, v, e)$ has length $n=g+6 h+8 v+8 e$. Let $m(P)$ be the number of codelines (including multiplicities) that contain point $P$. If $P \in l_{0}$, then $m(P)=g+2 h$, if $P \in E_{0} \backslash l_{0}$ then $m(P)=3 h+2 v$. If $P \in H_{0} \backslash E_{0}$ then $m(P)=e$ whereas points $P$ outside $H_{0}$ have $m(P)=v+e$. For each hyperplane $H$ let $m(H)=\sum_{P \in H} m(P)$. By double counting we obtain

$$
s(H)=(m(H)-n) / 2
$$

where $s(H)$ (the species of $H$ ) is the number of codelines contained in $H$. It follows that the numbers $n-s(H)$ are the nonzero weights of our code. The numbers $m(H)$ and $s(H)$ are easy to determine:

Lemma 2. If $l_{0} \not \subset H$ then $m(H)=g+8 h+12 v+12 e$.

If $l_{0} \subset H$ but $E_{0} \not \subset H$ then $m(H)=3 g+6 h+8 v+12 e$.

If $E_{0} \subset H \neq H_{0}$ then $m(H)=3 g+18 h+16 v+8 e$.

Finally $m\left(H_{0}\right)=3 g+18 h+8 v+8 e$.

Proof. This is a trivial calculation. In the first case above $H$ has one point of $l_{0}$, two further points in $E_{0}$, four further points in $H_{0}$ and finally 8 affine points for a grand total $m(H)=g+8 h+4 v+4 e+8(v+e)$. In the second case $H$ contains three points on $l_{0}$, no further point on $E_{0}$, four further points on $H_{0}$ and eight affine points: $m(H)=3 g+6 h+4 e+8(v+e)$. The remaining two cases are analogous. 
Our basic formula yields:

Corollary 1. The nonzero weights of the codewords of $C(g, h, v, e)$ are

$$
g+5 h+6(v+e), 6 h+8 v+6 e, 4 v+8 e, 8(v+e) .
$$

$C(g, h, v, e)$ is an $[g+6 h+8(v+e), 2.5, d]_{4}$-code where

$$
d=\operatorname{Min}\left(w_{1}=g+5 h+6(v+e), w_{2}=6 h+8 v+6 e, w_{3}=4 v+8 e\right) .
$$

We see that $C(2,0,1,2)$ is a $[26,2.5,20]$-code and $C(1,1,0,3)$ is a $[31,2.5,24]$ code. This completes the proof of Theorem 2. Lemma 1 yields

Corollary 2. A binary linear $[3 m, 5,2 e]_{2}$-code for $e<m-1$ exists if and only if the Griesmer bound $3(m-e) \geq e_{2}+e_{4}+e_{8}$ is satisfied.

\section{References}

[1] D. Bartoli, J. Bierbrauer, G. Faina, S. Marcugini, and F. Pambianco: The nonexistence of an additive quaternary [15, 5, 9]-code, Finite Fields and Their Applications 36 (2015), 29-40.

[2] J. Bierbrauer: Introduction to Coding Theory, Second Edition, Chapman and Hall/CRC Press, Fall 2016.

[3] J. Bierbrauer, Y. Edel, G. Faina, S. Marcugini, F. Pambianco: Short additive quaternary codes, IEEE IT Transactions 55 (2009), 952-954.

[4] J. Bierbrauer, G. Faina, M. Giulietti, S. Marcugini, and F. Pambianco: The geometry of quantum codes, Innovations in Incidence Geometry 6-7 (2009), 53-71.

[5] J. Bierbrauer, R.D. Fears, S. Marcugini, and F. Pambianco: The nonexistence of a $[[13,5,4]]$ quantum stabilizer code, arXiv:0908.1348, IEEE IT Transactions 57 (2011), 4788-4793.

[6] J. Bierbrauer, S. Marcugini, and F. Pambianco: A geometric nonexistence proof of an extremal additive code, Journal of Combinatorial Theory, Series A, 117 (2010), 128-137. 
[7] J. Bierbrauer, S. Marcugini, and F. Pambianco: Additive quaternary codes related to exceptional linear quaternary codes, IEEE IT Transactions 66 (2020), 273-277.

[8] A. Blokhuis and A. E. Brouwer, Small additive quaternary codes, European Journal of Combinatorics 25 (2004), 161-167.

[9] A. R. Calderbank, E. M. Rains, P. W. Shor, and N. J. A. Sloane: Quantum error correction via codes over $G F(4)$, IEEE Transactions on Information Theory 44 (1998), 1369-1387.

[10] N. Hamada and F. Tamari: Construction of optimal codes and optimal fractional factorial designs using linear programming, Annals of Discrete Mathematics 6 (1980), 175-188. 